\title{
Avaliação do impacto das universidades em suas comunidades
}

\author{
WAgNeR RAgI CURI FILHO ${ }^{1}$ \\ THOMAZ WOOD JUNIOR ${ }^{2}$ \\ ${ }^{1}$ Universidade Federal de Ouro Preto (UFOP) / Instituto de Ciências Exatas e Aplicadas, JoÃo MonleVade - MG, Brasil \\ ${ }^{2}$ FundaçÃo Getulio Vargas (FGV EAESP) / Escola de AdministraçÃo de EMPRESAS de SÃo PaUlo, SÃo PaUlo - SP, BRASIL
}

\section{Resumo}

Este artigo explora a questão do impacto das universidades sobre as comunidades nas quais estão instaladas. A partir de uma pesquisa bibliográfica, foram identificados e sistematizados três tipos de impacto: socioeconômico; científico-tecnológico e na cultura e imagem da região. Esses três tipos de impacto foram integrados em um modelo conceitual que poderá ser utilizado como base para um modelo prático voltado para avaliar o impacto de uma universidade sobre a região na qual se encontra. Além deles, o artigo também sugere passos para a implantação do modelo de avaliação de impacto. O texto contribui para o avanço dos estudos sobre o papel das universidades e pode ser útil para os gestores universitários na organização de projetos de avaliação de impacto.

Palavras-chave: Impacto de uma universidade na comunidade. Impacto socioeconômico. Impacto científico-tecnológico. Impacto na cultura e imagem da região.

\section{Evaluation of the impact of universities on their communities}

\begin{abstract}
This article investigates the impact of universities on the communities in which they are located. Based on bibliographical research, three types of impacts were identified and systematized: socioeconomic, scientific and technological, and on the culture and image of the region. These three types of impact have been integrated into a conceptual model that can be used as a basis for a practical model to be employed to assess the impact of a university on the region in which it is located. In addition to the three types of impact, the article also suggests steps for implementing the impact assessment model. This research contributes to the advancement of studies on the universities' role and can help university managers evaluate its impact on the community.
\end{abstract}

Keywords: University's impact on the community. Socioeconomic impact. Scientific and technological impact. Culture and image of the region.

\section{Evaluación del impacto de las universidades en sus comunidades}

\section{Resumen}

Este artículo explora el tema del impacto de las universidades en las comunidades en las que se encuentran. A partir de una investigación bibliográfica, se identificaron y sistematizaron tres tipos de impacto: socioeconómico; científico-tecnológico y en la cultura y la imagen de la región. Estos tres tipos de impacto se han integrado en un modelo conceptual que se puede utilizar como base para un modelo práctico que se empleará para evaluar el impacto de una universidad en la región en la que se encuentra. Además de los tres tipos de impacto, el artículo también sugiere pasos para la implementación del modelo de evaluación de impacto. El texto contribuye al avance de los estudios sobre el papel de las universidades y puede ser útil para los administradores universitarios en la organización de proyectos prácticos de evaluación de impacto.

Palabras clave: Impacto de una universidad en la comunidad. Impacto socioeconómico. Impacto científico-tecnológico. Impacto en la cultura e imagen de la región. 


\section{INTRODUÇÃO}

Friedrich von Humboldt (1767-1835) acreditava que a base e fim de qualquer sistema educacional era a formação de cidadãos, e defendia que somente a autonomia permitiria às universidades atingirem seus mais altos propósitos (Rohe, 2017). Vannevar Bush (1890-1974), no texto seminal 'Science, The Endless Frontier' (Bush, 1945) argumentou que o progresso científico é resultado da interação livre de intelectos livres: cientistas trabalhando em temas de sua escolha, definidos a partir de sua curiosidade para explorar o desconhecido e avançar as fronteiras da ciência.

Esses dois princípios - autonomia universitária e liberdade criativa - fundamentaram a institucionalização de sistemas de educação superior em muitos países, e foram validados pelo gigantesco progresso científico experimentado no século XX. No final do século XX, a ciência era um campo institucionalizado, globalizado e superespecializado. E a evolução continuava (e continua) em novas áreas de conhecimento e em domínios transdisciplinares. Com o desenvolvimento da ciência, cresceram também as demandas por investimentos e os orçamentos de pesquisa.

No entanto, a expansão começou a enfrentar limites orçamentários. A liberdade para criar começou a ser contraposta a necessidades sociais e econômicas, imperativos já antecipados por Bush (1945). Novas políticas de estado começaram a privilegiar uma visão de ciência estratégica, orientada para o fomento da competitividade (Gibbons, Limoges \& Scott, 2011). Com isso, o pêndulo entre a defesa da autonomia acadêmica e o imperativo de servir à sociedade está se movendo para a segunda base (Rohe, 2017). Este artigo se concentra em um aspecto relevante desse movimento: a questão do impacto das universidades sobre as comunidades nas quais se encontram.

De fato, as universidades podem contribuir para as comunidades que as hospedam (Silva, 2006). Elas podem gerar empregos para profissionais da região e proporcionar oportunidades de estudo para jovens locais (por exemplo, Drucker \& Goldstein, 2007; Johansen \& Arano, 2016). Seus projetos de pesquisa podem promover o desenvolvimento local. As universidades podem também fomentar a cultura e promover a imagem da região na qual estão situadas (Arbo \& Benneworth, 2007; Leten, Landoni \& Looy, 2014).

Por outro lado, vem crescendo o questionamento sobre o papel das universidades na sociedade (Leten, Landoni \& Looy, 2014; Musselin, 2013; Vieira, Bellen \& Fialho, 2006; Weber, 2010), levando algumas delas a buscar melhor comunicar os benefícios que geram para suas comunidades e para a sociedade em geral (Lendel, 2015; Marcovitch, 2019; Martin, 2012; May \& Perry, 2006).

No Brasil, esse fenômeno ganha especial interesse no âmbito das instituições públicas de ensino superior. Essas instituições sofreram notável expansão nas últimas décadas, porém continuam sob pressão para aumento e melhoria dos serviços prestados, em ensino, pesquisa e extensão, ao mesmo tempo em que sofrem pressões orçamentárias (Alves et al., 2015; Audy, 2017; Tarocco, Sesso, Esteves \& Kureski, 2014; Wanzinack \& Signorelli, 2014).

Deve-se destacar o papel dos rankings universitários que influenciam o comportamento das instituições. Eles são utilizados como instrumentos para uma análise sobre as variadas possibilidades de impacto de uma universidade na comunidade (Marcovitch, 2019).

Tal contexto levou ao surgimento de modelos para avaliação do impacto de instituições de ensino e pesquisa sobre suas comunidades (European Foundation for Management Development [EFMD], 2015). Entretanto, a maioria dos modelos criados parece se concentrar especialmente em dois aspectos: o impacto econômico e a capacidade de contribuição para o desenvolvimento tecnológico (Kantor \& Whalley, 2014; Scandura, 2016; Sen, 2011).

Diante disso e da provável inexistência de modelos integrados para avaliação do impacto de universidades, este artigo tem como objetivo contribuir para o desenvolvimento de um modelo conceitual amplo que possa ser utilizado como base para a construção de um modelo prático a ser aplicado à avaliação do impacto de uma universidade em sua comunidade. Para isso, foi realizada uma revisão bibliográfica, a qual identificou três tipos de impacto: socioeconômico; científico-tecnológico; e cultura e atratividade da região na qual a universidade está inserida. Para cada tipo de impacto foram definidos meios de impacto e sugeridos indicadores. 
Este artigo traz contribuições para a ciência e para a prática. O estudo contribui para os estudos sobre o novo papel das universidades (Huggins, Johnston \& Steffenson, 2008; Mccowan, 2016; Sleutjes, 1999) e avança a modelagem do impacto social das universidades, ao integrar as três dimensões encontradas na literatura, as quais haviam sido exploradas individualmente em trabalhos anteriores (Guerrero, Cunningham \& Urbano, 2015; Kantor \& Whalley, 2014; Popescu \& Corbos, 2012). Além disso, modelos desenvolvidos a partir do modelo proposto poderão ser utilizados, com adaptações às características de cada universidade e de cada região, por gestores universitários interessados em avaliar o impacto de suas instituições sobre as comunidades nas quais estão instaladas. É relevante frisar que este trabalho propõe um modelo preliminar que deve ser aprimorado e testado, de forma a subsidiar a construção de um modelo prático para aplicação à avaliação do impacto de uma universidade em sua comunidade.

Além dessa introdução, este trabalho conta com mais quatro seções. A segunda seção apresenta a pesquisa bibliográfica realizada para fundamentar o desenvolvimento do modelo. A terceira seção sistematiza os três tipos de impacto identificados na literatura e os integra no modelo de avaliação de impacto. A quarta seção propõe um processo de operacionalização do modelo de avaliação de impacto. A quinta seção apresenta comentários, indicando direções para futuros desenvolvimentos do modelo e futuras pesquisas.

\section{MÉTODOS}

Diante do objetivo de desenvolver um modelo conceitual (Crossan, Lane \& White, 1999) para a avaliação do impacto de uma universidade na comunidade, foi realizada uma revisão bibliográfica (Mayring, 2004). Inicialmente, buscou-se no trabalho de Mainardes, Alves e Raposo (2010) uma resposta para a questão de quem seriam os stakeholders de uma universidade. $O$ autor sugere uma lista de 21 diferentes tipos de stakeholders, sendo alguns específicos, tais como estudantes e professores, e outros mais genéricos, como a comunidade da região na qual a universidade está instalada. Considerando que as universidades podem impactar stakeholders tão distintos, buscaram-se artigos sobre os tipos de impactos que uma universidade pode gerar na comunidade local.

A coleta foi realizada em bases de artigos, tais como EBSCO, Scielo e Google Acadêmico, a partir das seguintes palavras-chave (em português e inglês): universidade, impacto e ambiente. Foram localizados 676 artigos, com data de publicação entre 1998 e 2018.

A partir dessa base, foi aplicado um filtro, de forma a selecionar textos que tratassem de impactos mais gerais, tais como: impacto nas indústrias, impacto na economia ou impacto na sustentabilidade da região. A seleção foi feita com base na leitura dos resumos e, quando isso não se mostrava suficiente, com base na leitura dos artigos completos. Resultaram desse processo 66 artigos.

Os artigos foram, então, integralmente analisados. Avaliando os objetivos desses artigos, emergiram três tipos de impacto, a partir dos quais os textos foram organizados. A Figura 1 representa os tipos de impacto encontrados. Além dos objetivos, foram identificadas, para cada artigo analisado integralmente, as variáveis que foram tratadas para medir o impacto da universidade. A elaboração do modelo proposto considerou, em sua estrutura, as variáveis identificadas nos artigos de forma a desenvolver um modelo de caráter integrador. 
Figura 1

Tipos de impacto

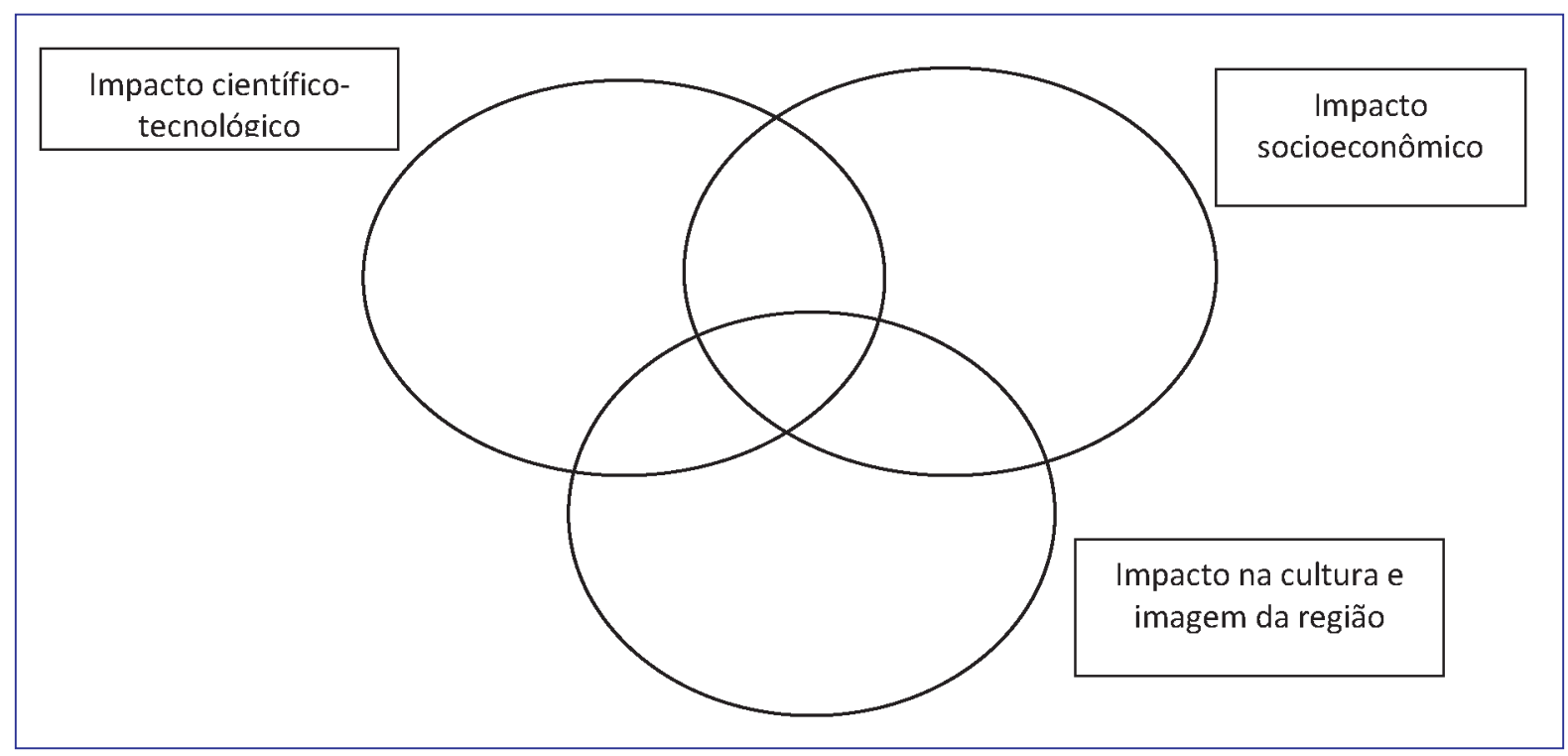

Fonte: Elaborada pelos autores.

O primeiro grupo de artigos analisados compreende 26 textos que tratam do impacto socioeconômico, seja por demandas da universidade (inputs) seja por efeitos da presença da universidade (outputs). O segundo grupo compreende 29 textos que tratam do impacto científico-tecnológico, com textos que tratam da relação entre universidade, empresa e governo; trabalhos que tratam do desenvolvimento de tecnologias comercializáveis; e estudos que abordam o papel das universidades na promoção de novos negócios. O terceiro grupo compreende 11 textos que tratam do impacto na cultura e imagem da região. Neste grupo estão os trabalhos que discutem a relação da universidade com os aspectos culturais do local ou que apresentam análises sobre como a universidade contribui para a formação da imagem da região.

Cabe observar que os três tipos de impacto identificados possuem aspectos que se sobrepõem. Por exemplo, alguns projetos de pesquisa podem resultar simultaneamente em impacto científico-tecnológico, socioeconômico e sobre a imagem da região. Portanto, alguns indicadores podem ser analisados, ora como parte de um tipo de impacto, ora como parte de outro tipo de impacto. Entretanto, para facilitar a identificação e análise, os indicadores que podem resultar em mais de um tipo de impacto foram inseridos, no modelo conceitual, apenas no tipo de impacto considerado principal.

A revisão bibliográfica permitiu também identificar, para cada tipo de impacto, os respectivos meios de impacto, ou seja, os diversos canais ou formas pelos quais a universidade gera aquele tipo de impacto.

\section{DESENVOLVIMENTO DO MODELO CONCEITUAL}

\section{Impacto socioeconômico}

A análise do referencial teórico sobre impacto socioeconômico revelou dois subgrupos de artigos. O primeiro subgrupo de artigos foca os outputs de uma universidade (Cox \& Taylor, 2006; Goldstein \& Drucker, 2006; Kureski \& Rolim, 2009; Lendel, 2015; Steinaker, 2005). Os outputs incluem geração de empregos, dispêndio financeiro, projetos de extensão e outras atividades.

Nesse subgrupo foram encontrados diversos artigos que utilizam a metodologia insumo-produto para comparar os gastos proporcionais da universidade em relação à economia da região estudada (Pastor, Pérez \& Guevara, 2012; Tarocco et al., 2014). O objetivo é identificar interdependências entre diferentes setores da economia (Miller \& Blair, 2009). Algumas universidades empregaram essa metodologia para avaliar seus respectivos impactos, como, por exemplo, a Universidade de Vancouver (Roslyn, 2013), a Universidade de Atenas (Payne, 2016) e a Universidade do Pacífico (Pogue, 2010). 
O segundo grupo de artigos foca os inputs de uma universidade (Cox \& Taylor, 2006; Kureski \& Rolim, 2009; Lendel, 2015). Os inputs incluem negócios diretos com a universidade, demandas por serviços públicos e demandas oriundas de políticas públicas (Verger, Curran \& Parcerisa, 2015).

Cabe ressaltar que a avaliação do impacto socioeconômico de uma universidade deve considerar o contexto no qual a instituição está situada, pois a capacidade de influência da universidade depende das características da região na qual ela se localiza (Huggins \& Johnston, 2009; Lazzeroni \& Piccaluga, 2015; Martin, 1998; Pastor et al., 2012). Por exemplo, universidades inseridas em grandes centros urbanos podem ter seu impacto socioeconômico diluído, enquanto que as universidades inseridas em pequenas cidades podem ter seu impacto socioeconômico ressaltado.

A Figura 2 sistematiza os meios possíveis de impacto socioeconômico de uma universidade, com respectivos indicadores, para a dimensão do impacto socioeconômico. Cada instituição interessada em avaliar seu impacto pode prover maior ou menor ênfase para cada meio de impacto, dependendo do objetivo da avaliação desejada.

Figura 2

Impacto socioeconômico de uma universidade

\begin{tabular}{|l|l|}
\hline Geração de empregos & $\begin{array}{l}\text { INDICADORES } \\
\text { - Número de empregos com alta qualificação } \\
\text { Dispêndio financeiro }\end{array}$ \\
\hline Projetos de extensão médio
\end{tabular}

Fonte: Elaborada pelos autores.

\section{Impacto científico-tecnológico}

A análise do referencial teórico sobre impacto científico-tecnológico revelou um corpo teórico que trata das relações entre universidade e indústria. Pode-se classificar tal corpo teórico em três subgrupos. O primeiro subgrupo contém trabalhos focando a relação entre universidade, empresa e governo (denominada tripla hélice). Seus autores argumentam que o impacto da universidade se dará a partir da promoção da inovação por meio das ações integradas desses três agentes (Doin \& Rosa, 2019; Etzkowitz \& Leydesdorff, 2000; Gomes \& Pereira, 2015; Ipiranga, Freitas \& Paiva, 2010; Johnson, 2008; Leydesdorff \& Meyer, 2003; Sutz, 2000;). 
O segundo subgrupo contém trabalhos que defendem uma agenda de pesquisa direcionada ao desenvolvimento de tecnologias comercializáveis, focando a relação direta entre universidade e indústria (Bramwell \& Wolfe, 2008; Curi, Daraio \& Llerena, 2012; Fernandes et al., 2010; Fisher, Atkinson-Grosjean \& House, 2001; Scandura, 2016) e aponta áreas de cooperação para tornar a relação entre universidade e indústria mais efetiva (Anderson, Daim \& Lavoie, 2007; Barnes \& Erkut, 2002).

O terceiro subgrupo contém trabalhos que tratam do papel da universidade na promoção da inovação e no desenvolvimento de novos negócios. Esses trabalhos focam a capacidade de as universidades fomentarem a inovação em suas regiões (Cowan \& Zinovyeva, 2013); e o engajamento de acadêmicos no desenvolvimento de novos empreendimentos (Muscio, Quaglione \& Ramaciotti, 2016; Rasmussen \& Borch, 2010). Alguns autores focam especificamente na contribuição das universidades para a formação de clusters (Gerolamo et al., 2008; May \& Perry, 2006).

A Figura 3 sistematiza os meios de impacto possíveis de uma universidade, com respectivos indicadores, para a dimensão do impacto científico-tecnológico. Como no caso anterior, cada instituição pode prover maior ou menor ênfase para cada meio de impacto, dependendo do objetivo da avaliação desejada.

Figura 3

Impacto científico-tecnológico de uma universidade

\begin{tabular}{|c|c|}
\hline MEIO DE IMPACTO & INDICADORES \\
\hline Capital intelectual & $\begin{array}{l}\text { - Número de estagiários e egressos } \\
\text { - Número de projetos de prestação de serviços } \\
\text { - Número de projetos de extensão voltados para organizações } \\
\text { - Número de projetos de pesquisa voltados para organizações } \\
\text { produtivas } \\
\text { - Existência de clusters influenciados pela universidade }\end{array}$ \\
\hline Políticas públicas & $\begin{array}{l}\text { - Participação da universidade em órgãos públicos e sociais que } \\
\text { contribuem para elaboração de políticas públicas }\end{array}$ \\
\hline Projetos de Pesquisa & $\begin{array}{l}\text { - Número de projetos de pesquisa voltados para demandas locais } \\
\text { - Produtos de pesquisas (número de patentes e/ou número de } \\
\text { publicações) }\end{array}$ \\
\hline Demandas por políticas públicas & $\begin{array}{l}\text { - Número de organizações produtivas locais que realizam projetos } \\
\text { com a universidade }\end{array}$ \\
\hline Projetos com empresas locais & $\begin{array}{l}\text { - Número de leis e políticas públicas que incentivem a realização } \\
\text { de projetos de pesquisa voltados para a comunidade }\end{array}$ \\
\hline
\end{tabular}

Fonte: Elaborada pelos autores.

\section{Impacto na cultura e na imagem da região}

A análise do referencial teórico sobre impacto na cultura e na imagem da região revelou um corpo teórico que pode ser classificado em dois subgrupos. O primeiro subgrupo de artigos trata do impacto da universidade nas artes e sua participação em eventos culturais locais. Estes trabalhos analisam a possibilidade de os eventos artísticos e culturais promoverem desenvolvimento econômico (Guetzkow, 2002; Wyk, Saayman \& Rossouw, 2013). Para Guetzkow (2002), por exemplo, destaca-se que, do ponto de vista individual, um evento artístico pode gerar empregos, além de estimular o autoconhecimento, enquanto, do ponto de vista de uma comunidade, pode contribuir para a economia e para a imagem da comunidade. Popescu e Corbos (2012), por sua vez, apresentam uma análise de como comunidades podem se estruturar a partir de eventos artísticos e culturais, apoiados por universidades. 
O segundo subgrupo de artigos pode ser caracterizado pelo debate sobre o papel da universidade no fomento de políticas e manifestações culturais. Arbo e Benneworth (2007), por exemplo, comentam o papel integrador que essas instituições podem ter sobre políticas locais. Complementarmente, Alshuwaikhat e Abubakar (2008), Hubbard (2008), Jain e Pant (2010) e Salmeron (2001) ressaltam a influência que uma universidade pode exercer sobre determinadas características culturais e da imagem de uma comunidade.

A Figura 4 sistematiza os meios de impacto possíveis de uma universidade, com respectivos indicadores, para a dimensão do impacto na cultura e imagem. Como nos casos anteriores, cada instituição pode prover maior ou menor ênfase para cada meio de impacto, dependendo do objetivo da avaliação desejada.

Figura 4

Impacto de uma universidade na cultura e na imagem da região

\begin{tabular}{|c|c|}
\hline MEIO DE IMPACTO & INDICADORES \\
\hline Ações de disseminação & $\begin{array}{l}\text { - Número de veiculações de áudio, imagem e texto da } \\
\text { universidade que mencionam a região } \\
\text { - Número de veiculações de áudio, imagem e texto sobre a } \\
\text { universidade que mencionam a região }\end{array}$ \\
\hline $\begin{array}{l}\text { Eventos culturais e sociais } \\
\text { promovidos pela universidade }\end{array}$ & $\begin{array}{l}\text { - Número de eventos culturais e sociais } \\
\text { - Número de participantes locais de eventos culturais e sociais } \\
\text { - Número de participantes externos de eventos culturais e sociais }\end{array}$ \\
\hline $\begin{array}{l}\text { Políticas públicas voltadas para } \\
\text { promoção da cultura e da } \\
\text { imagem da região }\end{array}$ & $\begin{array}{l}\text { - Participações da universidade no aperfeiçoamento de políticas } \\
\text { públicas voltadas para a cultura e imagem da região } \\
\text { - Número de projetos da universidade que envolvam setores } \\
\text { públicos e a elaboração de políticas públicas }\end{array}$ \\
\hline
\end{tabular}

Fonte: Elaborada pelos autores.

\section{Modelo conceitual integrado de avaliação do impacto}

A integração dos três modelos anteriores resulta no modelo conceitual de avaliação de impacto da universidade na região na qual se localiza. A Figura 5 representa esse modelo. Cabe uma vez mais notar que o modelo deve ser visto como uma matriz geral, que poderá ser utilizada para o desenvolvimento de um modelo de aplicação prática, a ser adaptado para cada universidade e cada comunidade. Da mesma forma, poder-se-á trabalhar na adequação dos indicadores a serem utilizados em cada caso. 
Figura 5

Modelo conceitual de avaliação de impacto: tipos e meios de impacto

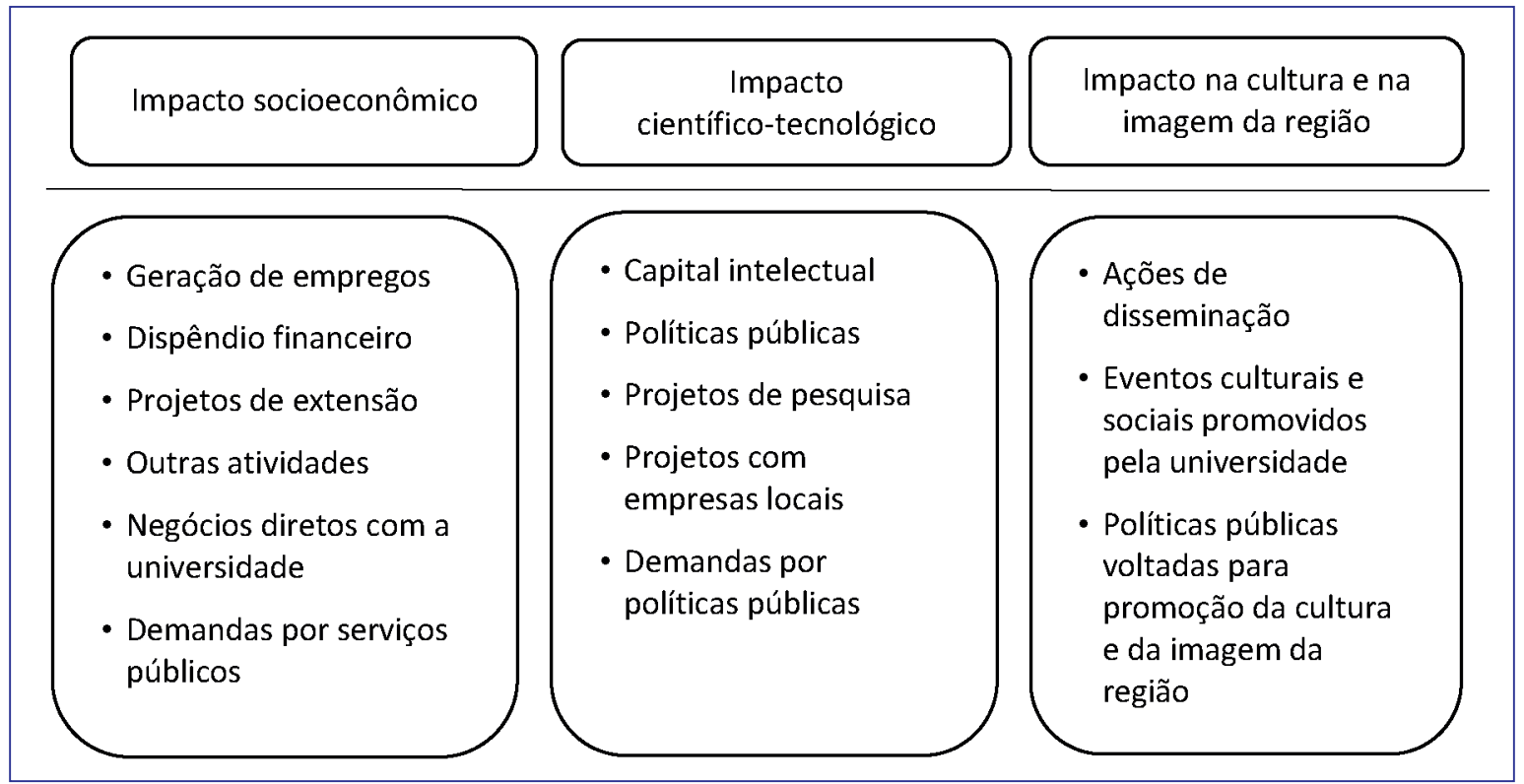

Fonte: Elaborada pelos autores.

\section{Contribuições brasileiras ao debate}

Neste ponto do texto, é conveniente destacar algumas contribuições brasileiras ao tema do impacto das universidades em suas comunidades. No Brasil, o tema do impacto social das universidades pode ser relacionado a discussões sobre: 1. indicadores de desempenho acadêmico; 2 . os sistemas de avaliação; e 3. os rankings universitários. De forma mais ampla, pode ser igualmente relacionado a reflexões sobre autonomia universitária e à relação entre universidade e sociedade. Sem a pretensão de retratar ou sumarizar o rico debate sobre essas questões que acontecem no país, são destacados a seguir alguns autores e trabalhos mais diretamente relacionados ao presente estudo.

Uma primeira questão, que caracteriza as reflexões brasileiras e apresenta direta relação com este artigo é a questão dos outputs, ou seja, os efeitos que a universidade gera sobre a comunidade. A identificação de outputs pode ser encontrada em trabalhos que se concentram no debate sobre indicadores utilizados na elaboração de rankings universitários e em trabalhos cujo alvo é a questão da avaliação de programas de pós-graduação. Por exemplo, Marcovitch e Axel-Berg (2019) observam a necessidade de utilizar indicadores quantitativos e qualitativos, e alertam que as instituições devem se preocupar com o eventual mau uso dos indicadores na "criação de políticas, avaliações, rankings e decisões de promoção e contratação de docentes" (Marcovitch \& Axel-Berg, 2019, p. 128).

Ranieri (2019), por sua vez, indica especificamente os temas que devem ser considerados, ao se buscar os outputs de uma universidade: pesquisa e produção acadêmica; impacto social da produção acadêmica; impacto das atividades de extensão; e impacto profissional dos egressos. De forma similar, Planeta et al. (2019) indicam as seguintes dimensões para medição do impacto social: econômica/financeira; socioeducacional; inovação e empreendedorismo; intelectual, cultura e bem-estar; e reputação. De fato, o modelo proposto pelos autores aproxima-se daquele proposto no presente estudo. A tendência de valorização do impacto social pode também ser constatada por meio da observação da evolução do sistema da Coordenação de Aperfeiçoamento de Pessoal de Nível Superior (CAPES) do Brasil (Barata, 2019; Gheno et al., 2019; Ministério da Educação, 2018).

Finalmente, vale ressaltar o debate sobre a transformação do conceito de autonomia universitária encontrado em Muzy e Drugowich (2018) e Balbachevsky e Kohtamäki (2019). Apesar de ter havido um aumento do controle externo, as universidades têm autonomia para tomar decisões sobre muitos aspectos da vida acadêmica. Por outro lado, o que se produz na universidade deve responder às demandas sociais. Por isso, mecanismos externos e internos de avaliação devem ser coerentes entre si. Dessa forma, as universidades devem utilizar os mecanismos externos e internos como instrumentos de gestão para que se amplie o impacto positivo na comunidade. 


\section{PROCESSO PARA OPERACIONALIZAÇÃO DA AVALIAÇÃO DE IMPACTO}

Além do modelo de avaliação de impacto, propõe-se, de forma complementar, um processo para operacionalização da avaliação de impacto. O objetivo do processo é facilitar a implantação, visando maximizar os impactos positivos e minimizar eventuais impactos negativos da universidade em sua comunidade local. Esse modelo deve ser implantado em seis fases, conforme indicado na Figura 6.

Figura 6

Modelo de operacionalização da avaliação de impacto: fases e ações principais

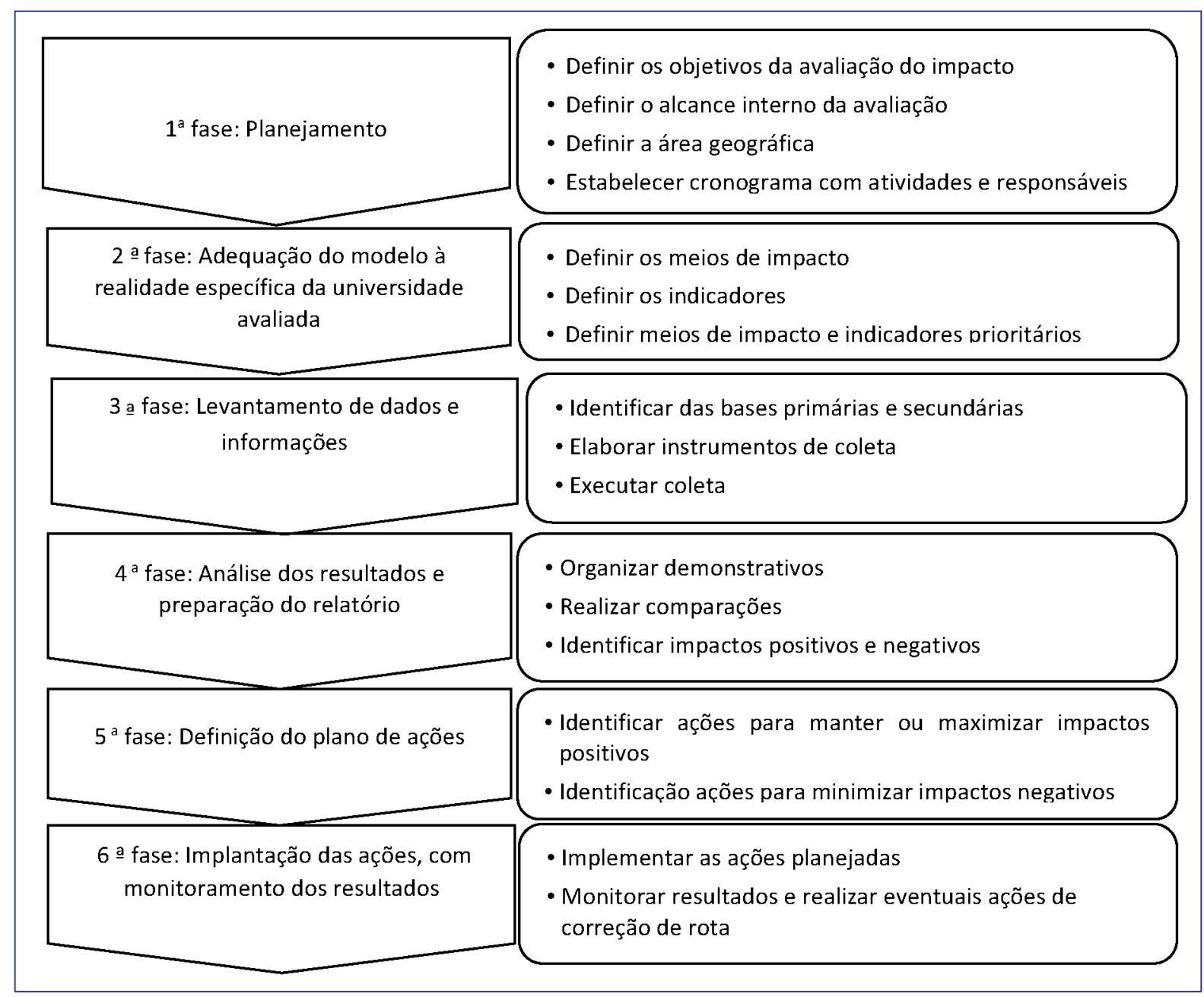

Fonte: Elaborada pelos autores.

A primeira fase destina-se principalmente à definição dos objetivos e do escopo do projeto. Os objetivos podem variar significativamente conforme o contexto e a universidade. Por exemplo, uma instituição tradicional e já estabelecida poderá estabelecer como objetivo principal aumentar seu impacto cultural sobre a comunidade na qual está localizada, contribuindo para o fortalecimento da imagem da região; outra instituição, jovem e em processo de expansão, poderá definir como meta aumentar seu impacto científico-tecnológico, contribuindo assim para consolidar o nascente parque industrial da região na qual foi instalada; e uma terceira instituição, enfrentando dificuldades de financiamento, poderá ter como foco demonstrar seu impacto em todas as dimensões diante de seus públicos de interesse, de forma a melhorar relacionamentos e facilitar a obtenção de recursos para pesquisas e outras atividades. 
A primeira fase destina-se também a definir o alcance interno da avaliação, a qual poderá restringir-se a uma única unidade (por exemplo, uma faculdade), um conjunto de faculdades ou toda a universidade.

A primeira fase destina-se, finalmente, a definir a área que será objeto da avaliação. Algumas instituições podem restringir seu foco à cidade na qual estão instaladas; enquanto outras podem ampliar seu foco para conjuntos de cidades, um estado, ou ainda uma região inteira.

A segunda fase destina-se a reconstruir o modelo de acordo com as definições da primeira fase. Recomenda-se que a reconstrução seja feita de "cima para baixo", ou seja, primeiro, deve-se definir os tipos de impacto a serem avaliados: socioeconômico e/ou científico-tecnológico e/ou na cultura e na imagem da região; em seguida, deve-se definir os meios de impacto, eliminando eventualmente alguns daqueles listados na Figura 5 e acrescentando eventualmente outros; finalmente, deve-se rever os indicadores para os meios de impacto escolhidos, eliminando alguns daqueles listados nas Figuras 2, 3 e 4, e acrescentando outros. Recomenda-se também escolher, nessa fase, os meios de impacto e indicadores prioritários, sendo considerados como tal aqueles mais diretamente alinhados aos objetivos da avaliação.

A terceira fase tem caráter técnico e operacional. Destina-se a definir como será feita a coleta, possivelmente combinando obtenção de dados primários e secundários, e executando-a. Deve-se observar que parte dos dados e informações a serem coletados pode ser de fácil acesso, constando de documentos já existentes, enquanto outra parte exigirá um esforço específico de obtenção. Além disso, pode-se supor que haverá coleta tanto de dados quantitativos quanto de informações qualitativas.

A quarta fase compreende a compilação e análise dos dados e informações obtidos na fase anterior. Além de preparar quadros demonstrativos dos impactos, deve-se nessa fase comparar resultados (por exemplo, com referências externas, e/ou entre unidades, e/ou com resultados anteriores). Deve-se também identificar impactos positivos e negativos da universidade sobre a comunidade local.

A quinta fase toma como base a fase anterior. A partir dos impactos positivos, devem-se identificar ações para sua conservação e, idealmente, maximizá-los. A partir dos impactos negativos encontrados, devem-se identificar ações para minimizá-los e, idealmente, suprimi-los.

A sexta fase corresponde à implementação das ações definidas na quinta fase. Além da execução, devem-se monitorar continuamente os resultados, de forma a garantir que estejam de acordo com o planejado, realizando, quando necessário, ações de correção de rota.

\section{CONCLUSÃO}

Este artigo apresentou um modelo conceitual de avaliação do impacto de uma universidade em sua comunidade local. Tal modelo foi construído com base na literatura existente, a qual indica três tipos de impacto: socioeconômico, científico-tecnológico, e na cultura e imagem da região. Além do modelo de avaliação de impacto, este trabalho apresentou um segundo modelo, visando a operacionalização do modelo de avaliação de impacto.

Considerando o contexto brasileiro, o artigo chama a atenção para dimensões presentes na literatura científica, porém não consideradas nos sistemas nacionais de avaliação. Esses sistemas privilegiam três dimensões de atividades da universidade: ensino, pesquisa e extensão (Weber, 2010; Pinto, Mello \& Melo, 2016). Os sistemas locais utilizam listas amplas de critérios e parâmetros para avaliar instituições e programas. Eles diferem do sistema aqui discutido em pelo menos dois aspectos. O primeiro aspecto é a finalidade: os sistemas brasileiros destinam-se a garantir e fomentar a qualidade das instituições e dos programas de ensino e pesquisa do país, enquanto o sistema proposto nesse artigo objetiva avaliar e fomentar o impacto positivo das instituições em suas respectivas comunidades ou regiões. O segundo aspecto é a forma de aplicação: os sistemas locais empregam padrões únicos para avaliar instituições com características diferentes, enquanto o sistema proposto, embora possa utilizar dimensões e critérios comuns, pressupõe uma aplicação customizada à cada contexto específico. Assim, o modelo de avaliação de impacto aqui apresentado pode ser visto como um complemento, não substituto, dos sistemas existentes. 
Deve-se, finalmente, observar que este trabalho constitui um esforço inicial, cujo produto poderá ser enriquecido. O modelo conceitual proposto não constitui um sistema pronto para o uso. Ele deve ser validado empiricamente, de forma que possa ser utilizado como ferramenta administrativa por gestores de instituições de ensino e pesquisa. Além disso, futuros desenvolvimentos deverão focar a questão da operacionalização da avaliação, a qual encerra desafios metodológicos complexos.

Em relação a futuras pesquisas, uma primeira possibilidade é realizar estudos de caso, aplicando o modelo a diferentes universidades, de forma a avaliar criticamente seu potencial e aperfeiçoá-lo. Uma segunda possibilidade é realizar estudos comparativos de casos, entre diferentes unidades e/ou diferentes universidades, de forma a avaliar como contextos distintos podem ser tratados. Uma terceira possibilidade é realizar estudos longitudinais, observando como uma universidade, ou uma unidade dentro de uma universidade, evolui ao longo do tempo.

\section{AGRADECIMENTOS}

Este artigo teve origem na tese de Wagner Curi. O autor agradece à CAPES, pelo apoio à realização de seu doutorado (Dinter). Os autores agradecem aos avaliadores anônimos por suas sugestões. 


\section{REFERÊNCIAS}

Alshuwaikhat, H. M., \& Abubakar, I. (2008). An integrated approach to achieving campus sustainability: assessment of the current campus environmental management practices. Journal of cleaner production, 16(16), 1777-1785.

Alves, J., Carvalho, L., Carvalho, R., Correia, F., Cunha, J. ... Nicolau, A. (2015). The impact of polytechnic institutes on the local economy. Tertiary Education and Management, 21(2), 81-98.

Anderson, T. R., Daim, T. U., \& Lavoie, F. F. (2007). Measuring the efficiency of university technology transfer. Technovation, 27(5), 306-318.

Arbo, P., \& Benneworth, P. (2007). Understanding the regional contribution of higher education institutions: A literature review. Recuperado de http://dx.doi.org/10.1787/161208155312

Audy, J. (2017). A inovação, o desenvolvimento e o papel da Universidade. Estudos avançados, 31(90), 75-87.

Balbachevsky, E., \& Kohtamäki, V. (2019). Autonomia e Governança Universitária: uma Abordagem Comparativa USP (Brasil) e UTA (Finlândia). In J. Marcovitch (Org.), Repensar a Universidade: Impactos para a Sociedade. São Paulo, SP: Com-Arte; Fapesp.

Barata, R. (2019). Mudanças necessárias na avaliação da pós-graduação brasileira. Interface - Comunicação, Saúde, Educação, 23, e180635. Recuperado de ttps://doi.org/10.1590/interface.180635

Bramwell, A., \& Wolfe, D. A. (2008). Universities and regional economic development: The entrepreneurial University of Waterloo. Research policy, 37(8), 1175-1187.

Barnes, T., Pashby, I., \& Gibbons, A. (2002). Effective universityindustry interaction: A multi-case evaluation of collaborative R\&D projects. European Management Journal, 20(3), 272-285.

Bush, V. (1945). Science, the Endless Frontier: A Report to the President. Washington, DC: U.S. Recuperado de https://archive.org/stream/ scienceendlessfro0unit/scienceendlessfro0unit_djvu.txt

Cowan, R., \& Zinovyeva, N. (2013). University effects on regional innovation. Research Policy, 42(3), 788-800.

Cox, S., \& Taylor, J. (2006). The impact of a business school on regional economic development: a case study. Local Economy, 21(2), 117-135.

Crossan, M. M., Lane, H. W., \& White, R. E. (1999). An organizational learning framework: From intuition to institution. Academy of management review, 24(3), 522-537.

Curi, C., Daraio, C., \& Llerena, P. (2012). University technology transfer: how (in) efficient are French universities?. Cambridge Journal of Economics, 36(3), 629-654.

Doin, T., \& Rosa, A. R. (2019). Interação Universidade-EmpresaGoverno: o caso do Programa de Cooperação Educacional para Transferência de Conhecimento Brasil-Cingapura. Cadernos EBAPE.BR, 17(4), 940-958.

Drucker, J., \& Goldstein, H. (2007). Assessing the regional economic development impacts of universities: A review of current approaches. International regional science review, 30(1), 20-46.
Erkut, E. (2002). Measuring Canadian business school research output and impact. Canadian Journal of Administrative Sciences/ Revue Canadienne des Sciences de l'Administration, 19(2), 97-123.

Etzkowitz, H., \& Leydesdorff, L. (2000). The dynamics of innovation: from National Systems and "Mode 2" to a Triple Helix of universityindustry-government relations. Research policy, 29(2), 109-123.

European Foundation for Management Development. (2015). BSIS Process Guideline. Recuperado de http://www.efmdglobal.org/images/ download/BSIS/BSIS\%20Process\%20Guidelines-141204-PRINT.pdf

Fernandes, A. C., Souza, B. C., Silva, A. S., Suzigan, W., Chaves, C. V. ... Albuquerque, E. (2010). Academy-industry links in Brazil: evidence about channels and benefits for firms and researchers. Science and Public Policy, 37(7), 485-498.

Fisher, D., Atkinson-Grosjean, J., \& House, D. (2001). Changes in academy/industry/state relations in Canada: The creation and development of the networks of centres of excellence. Minerva, 39(3), 299-325.

Gerolamo, M. C., Carpinetti, L. C. R., Fleschutz, T., \& Seliger, G. (2008). Clusters e redes de cooperação de pequenas e médias empresas: observatório europeu, caso alemão e contribuições ao caso brasileiro. Gestão \& Produção, 15(2), 351-365.

Gheno, E. M., Guaragna, R. M., Mata, L. F. S., Duarte, L. F., Souza, D. O. ... Calabró, L. (2019). Sistema de avaliação da CAPES: indicadores e procedimentos de monitoramento e avaliação de desempenho. Em Questão, 25(3), 184-213.

Gibbons, M., Limoges, C., \& Scott, P. (2011). Revisiting Mode 2 at Noors Slott. Prometheus, 29(4), 361-372.

Goldstein, H., \& Drucker, J. (2006). The economic development impacts of universities on regions: Do size and distance matter?. Economic development quarterly, 20(1), 22-43.

Gomes, M. A. S., \& Pereira, F. E. C. (2015). Hélice Tríplice: Um ensaio teórico sobre a relação Universidade-Empresa-Governo em busca da inovação. International Journal of Knowledge Engineering and Management (IJKEM), 4(8), 136-155.

Guerrero, M., Cunningham, J. A., \& Urbano, D. (2015). Economic impact of entrepreneurial universities' activities: An exploratory study of the United Kingdom. Research Policy, 44(3), 748-764.

Guetzkow, J. (2002). How the arts impact communities: an introduction to the literature on arts impact studies (Working Paper Series, 20). Princeton, NJ: Centre for Arts and Cultural Policy Studies.

Hubbard, P. (2008). Regulating the social impacts of studentification: a Loughborough case study. Environment and Planning A, 40(2), 323-341.

Huggins, R., \& Johnston, A. (2009). The economic and innovation contribution of universities: a regional perspective. Environment and Planning C: Government and Policy, 27(6), 1088-1106.

Huggins, R., Johnston, A., \& Steffenson, R. (2008). Universities, knowledge networks and regional policy. Cambridge Journal of Regions, Economy and Society, 1(2), 321-340. 
Ipiranga, A. S. R., Freitas, A. A. F. D., \& Paiva, T. A. (2010). O empreendedorismo acadêmico no contexto da interação universidadeempresa-governo. Cadernos EBAPE.BR, 8(4), 676-693.

Jain, S., \& Pant, P. (2010). Environmental management systems for educational institutions. International Journal of Sustainability in Higher Education 11(3), 236-249.

Johansen, T., \& Arano, K. (2016). The long-run economic impact of an institution of higher education: Estimating the human capital contribution. Economic Development Quarterly, 30(3), 203-214.

Johnson, W. H. (2008). Roles, resources and benefits of intermediate organizations supporting triple helix collaborative R\&D: The case of Precarn. Technovation, 28(8), 495-505.

Kantor, S., \& Whalley, A. (2014). Knowledge spillovers from research universities: evidence from endowment value shocks. Review of Economics and Statistics, 96(1), 171-188.

Kureski, R., \& Rolim, C. (2009). Impacto econômico de curto prazo das universidades federais na economia brasileira. Revista Paranaense de Desenvolvimento, 117, 29-51.

Lazzeroni, M., \& Piccaluga, A. (2015). Beyond 'town and gown': the role of the university in small and medium-sized cities. Industry and Higher Education, 29(1), 11-23.

Lendel, I. (2010). The impact of research universities on regional economies: The concept of university products. Economic Development Quarterly, 24(3), 210-230.

Leten, B., Landoni, P., \& Van Looy, B. (2014). Science or graduates: How do firms benefit from the proximity of universities?. Research Policy, 43(8), 1398-1412.

Leydesdorff, L., \& Meyer, M. (2003). The Triple Helix of universityindustry-government relations. Scientometrics, 58(2), 191-203.

Mainardes, E. W., Alves, H., \& Raposo, M. (2010). An exploratory research on the stakeholders of a university. Journal of Management and Strategy, 1(1), 76.

Marcovitch, J. (2019). A Universidade em 2022. Estudos Avançados, 33(95), 7-18.

Marcovitch, J., \& AXEL-BERG, J. (2019). Consenso para a Excelência Acadêmica. In: Marcovitch, J. (Org). Repensar a Universidade: Impactos para a Sociedade. São Paulo, SP: Com-Arte; Fapesp.

Martin, B. R. (2012). Are universities and university research under threat? Towards an evolutionary model of university speciation. Cambridge journal of economics, 36(3), 543-565.

Martin, F. (1998). The economic impact of Canadian university R\&D. Research Policy, 27(7), 677-687.

May, T., \& Perry 1, B. (2006). Cities, knowledge and universities: Transformations in the image of the intangible. Social Epistemology, 20(3-4), 259-282.

Mayring, P. (2004). Qualitative content analysis. A companion to qualitative research, 4(1), 159-176.

McCowan, T. (2016). Universities and the post-2015 development agenda: an analytical framework. Higher Education, 72(4), 505-523.

Miller, R. E., \& Blair, P. D. (2009). Input-output analysis: foundations and extensions. Cambridge, UK: Cambridge University.
Ministério da Educação. (2018). Proposta de Aprimoramento do Modelo de Avaliação da PG. Documento Final da Comissão Nacional de Acompanhamento do PNPG 2011-2020. Brasília, DF. Recuperado de https://www.capes.gov.br/images/stories/download/conselhosuperior/18102018_PNPG_CS_Avaliacao_Final_CS_FINAL_17_55.pdf

Muscio, A., Quaglione, D., \& Ramaciotti, L. (2016). The effects of university rules on spinoff creation: The case of academia in Italy. Research Policy, 45(7), 1386-1396.

Musselin, C. (2013). How peer review empowers the academic profession and university managers: Changes in relationships between the state, universities and the professoriate. Research Policy, 42(5), 1165-1173.

Muzy, J. R., \& Drugowich, P. A. (2018). Os Desafios da Autonomia Universitária: História Recente da USP. Jundiai, SP: Paco.

Pastor, J. M., Pérez, F., \& De Guevara, J. F. (2013). Measuring the local economic impact of universities: An approach that considers uncertainty. Higher education, 65(5), 539-564.

Payne Consulting. (2016). Athens State University: Economics Impacts. Recuperado de http://www.athens.edu/pdfs/about/economicimpact.pdf?30255e

Pinto, R. S., Mello, S., \& Melo, P. A. (2016). Meta-avaliação: uma década do Processo de Avaliação Institucional do SINAES. Avaliação: Revista da Avaliação da Educação Superior, 21(1), 89-108.

Planeta, C. S., Marques, A.C., Bueno, G.W., Luque, C.A., Hashimoto, F., \& Gontijo, J. (2019). Impacto Social das Universidades. In: Marcovitch, J. (Org) Repensar a Universidade: Impactos para a Sociedade. São Paulo, SP: Com-Arte; Fapesp.

Pogue, T. E. (2010). University of the Pacific: community impact. 2010. Recuperado de http://www.pacific.edu/Documents/marketing/ community-impact-report.pdf

Popescu, R. I., \& Corbos, R. A. (2012). The role of festivals and cultural events in the strategic development of cities. Recommendations for urban areas in Romania. Informatica Economica, 16(4), 19.

Ranieri, N. (2019). O Direito e suas Métricas. In: Marcovitch, J. (Org) Repensar a Universidade: Impactos para a Sociedade. São Paulo, SP: Com-Arte; Fapesp.

Rasmussen, E., \& Borch, O. J. (2010). University capabilities in facilitating entrepreneurship: A longitudinal study of spin-off ventures at midrange universities. Research policy, 39(5), 602-612.

Rohe, W. (2017). The Contract between Society and Science: Changes and Challenges. Social Research: An International Quarterly, 84(3), 739-757.

Roslyn, K. (2013). Vancouver Island University: Economic Impact Analyis \& Community Engagement. Recuperado de https://www2. viu.ca/impact/documents/VIU-Impact-Report.pdf

Salmeron, R. A. (2001). Universidade pública e identidade cultural. Tempo social, 13(1), 9-26.

Scandura, A. (2016). University-industry collaboration and firms' R\&D effort. Research Policy, 45(9), 1907-1922.

Sen, A. (2011). Local income and employment impact of universities: the case of Izmir University of Economics. Journal of Applied Economics and Business Research, 1(1), 25-42. 
Silva, F. L. (2006). Universidade: a idéia e a história. Estudos avançados, 20(56), 191-202.

Sleutjes, M. H. S. C. (1999). Refletindo sobre os três pilares de sustentação das universidades: ensino-pesquisa-extensão. Revista de Administração Pública, 33(3), 99-101.

Steinacker, A. (2005). The economic effect of urban colleges on their surrounding communities. Urban Studies, 42(7), 1161-1175.

Sutz, J. (2000). The university-industry-government relations in Latin America. Research policy, 29(2), 279-290.

Tarocco, J., Filho, Sesso, U. A., Filho, Esteves, E. Z. G., \& Kureski, R. (2014). Impacto econômico de curto prazo da Universidade Estadual de Londrina. Economia \& Região, 2(1), 83-103.

Van Wyk, L., Saayman, M., \& Rossouw, R. (2013). Economic impact of the Klein Karoo National Arts Festival: investigating the application of different models. Journal of Economic and Financial Sciences, 6(1), 129-152.

Verger, A., Curran, M., \& Parcerisa, L. (2015). La trayectoria de una reforma educativa global: el caso de la nueva gestión pública en el sistema educativo catalán. Educação \& Sociedade, 36(132), 675-697.

Vieira, E. M. F., Van Bellen, H. M., \& Fialho, F. A. P. (2006). Universidade em tempo de mudança. Cadernos EBAPE.BR, 4(3), 01-07.

Wanzinack, C., \& Signorelli, M. C. (2014). Expansão do ensino superior federal e desenvolvimento regional: o caso da Universidade Federal do Paraná no litoral paranaense. Revista Gestão Universitária na América Latina-GUAL, 7(2), 286-307.

Weber, S. (2010). Avaliação e regulação da educação superior: conquistas e impasses. Educação \& Sociedade, 31(113), 1247-1269.

Wagner Ragi Curi Filho

ORCID: https://orcid.org/0000-0002-8420-5710

Doutor em Administração pela Fundação Getulio Vargas (FGV EAESP); Professor Adjunto do Departamento de Engenharia de Produção da Universidade Federal de Ouro Preto (UFOP). E-mail: wagner@ufop.edu.br

Thomaz Wood Junior ORCID: https://orcid.org/0000-0002-5610-4699

Doutor em Administração pela Fundação Getulio Vargas (FGV EAESP); Professor Titular da Fundação Getulio Vargas (FGV EAESP).

E-mail: thomaz.wood@fgv.br 